\title{
Sudden Cardiac Death Caused by Cardiac Small Vessel Vasculitis after COVID-19 Vaccination (BNT162b2 nCov-19): A Case Report
}

Sohyung Park ${ }^{1}$, Min Kyoung Kim², Kanghyun Baek ${ }^{2}$

\author{
${ }^{1}$ Department of Forensic \\ Investigation, National Forensic \\ Service, Wonju, Korea, ${ }^{2}$ Department \\ of Forensic Medicine, National \\ Forensic Service Seoul Institute, \\ Seoul, Korea
}

Received: October 30, 2021 Revised: November 15, 2021 Accepted: November 23, 2021

\section{Correspondence to}

Sohyung Park

Department of Forensic Medicine, National Forensic Service, 139 Jiyang-ro, Yangcheon-gu, Seoul 08036, Korea

Tel: +82-2-2600-4614

Fax: $+82-2-2600-4828$

E-mail: zzeva@korea.kr
This study presents a case of sudden cardiac death due to cardiac small vessel vasculitis after coronavirus disease 2019 (COVID-19) mRNA vaccination (BNT162b2). The deceased was a 76-year-old woman with diabetes mellitus (DM) and chronic hypertension (HTN), who experienced generalized pain for a month after the first dose of vaccination, and unexpectedly died. Postmortem examination revealed small vessel vasculitis in the heart, lungs and vaccinated site of the left arm. These features were similar to those observed in a previously reported case of a patient with COVID-19 and cardiac endotheliitis and multisystem inflammatory syndrome. In addition, DM and HTN may contribute to vaccine-induced immunologic changes and vascular dysfunction. However, further studies with additional cases are needed.

Key Words: COVID-19 vaccines; Vasculitis

\section{Introduction}

The rate of vaccination is increasing as the coronavirus disease 2019 (COVID-19) pandemic continues to evolve with additional waves. COVID-19 mRNA vaccine (BNT162b2) is known to be mostly safe [1]. Various adverse events have been reported after COVID-19 vaccination, including myocarditis, lymphadenopathy, appendicitis, herpes zoster infection, pericarditis, arrhythmia, deep vein thrombosis, pulmonary embolism, myocardial infarction, intracranial hemorrhage, and thrombocytopenia [2]. However, an unexpected fatal case occurred recently after COVID-19 vaccination
(BNT162b2) which cannot be attributed to such known adverse events after vaccination. Hence, the postmortem findings of this case are presented with a review of the literature.

\section{Case Report}

The deceased was a 76-years-old woman found unresponsive in the early morning during hospitalization. The deceased had been experiencing pain on the upper body and arms for a month after the first dose of COVID-19 mRNA (BNT162b2) vaccination. The pain began in the evening on the day of vaccination, and 
the symptoms did not improve. She visited a university hospital two weeks after vaccination (13 days prior to death). Further evaluation was performed to determine the source of persistent pain of the body; however, she was ultimately discharged without a clear diagnosis. A real-time polymerase chain reaction test for severe acute respiratory syndrome coronavirus 2 was performed in the hospital, and the result was negative. Increased C-reactive protein levels (CRP; $4.40 \mathrm{ng} / \mathrm{mL}$; reference range not included) were noted on the medial record. One day prior to her death (four weeks after vaccination), the deceased called 119 (emergency number in Korea) for emergency medical assistance due to aggravating pain and mild confusion. The deceased was immediately transferred and admitted to another hospital. The next morning, the deceased was found unresponsive, and eventually died. The results of laboratory tests revealed elevated CRP levels (14.4 ng/mL: reference range $<0.3 \mathrm{mg} / \mathrm{dL}$ ). The deceased had a medical history of chronic hypertension, diabetes mellitus, hyperlipidemia, degenerative osteoarthritis of the both knee joints, and osteoporosis. This case was referred to the authors' institute because it was an unexpected death after vaccination and there was medicolegal dispute on the cause of death and treatment for the deceased.

On external examination, the deceased appeared obese and clean. The deceased's body mass index was $32.8 \mathrm{~kg} / \mathrm{m}^{2}$ (height, $160 \mathrm{~cm}$; and weight, $84 \mathrm{~kg}$ ). No injury was identified, and other findings were unremarkable. On internal examination, the heart weighed $440 \mathrm{~g}$ and cardiomegaly was observed. The circumferences of the tricuspid, pulmonary, mitral, and pulmonary valves were $13.2 \mathrm{~cm}, 8.5 \mathrm{~cm}, 10.5 \mathrm{~cm}$, and 7.0 $\mathrm{cm}$, respectively. The both ventricles were moderately dilated. The thicknesses of the left and right ventricular walls, and septum were $1.3 \mathrm{~cm}, 0.3 \mathrm{~cm}$, and $1.1 \mathrm{~cm}$, respectively. Mild stenosis (approximately 10\%) was observed in the left descending artery, but the heart was otherwise unremarkable. Both lungs and other internal organs were grossly unremarkable, as was the vaccinated site in the left upper arm. Two tiny nodules were identified in the right lobe of the thyroid gland.

On microscopic examination, most small arteries in the myocardium of both ventricles and sinoatrial

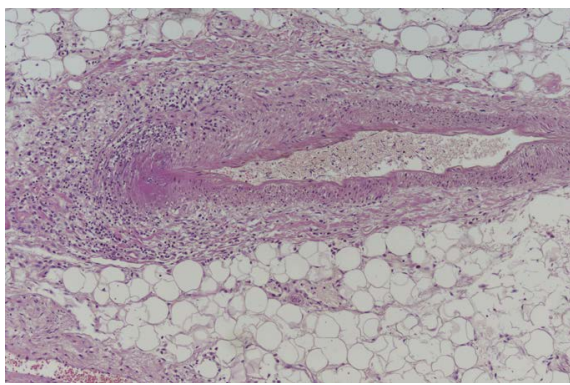

A

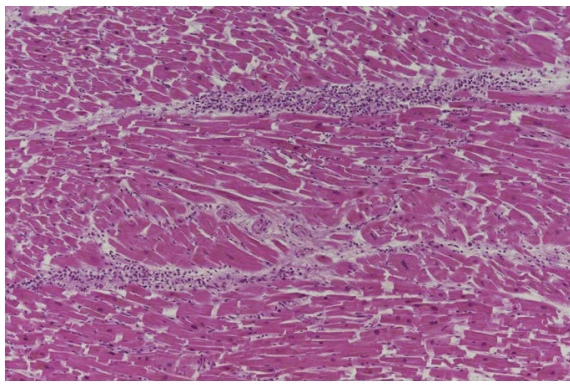

D

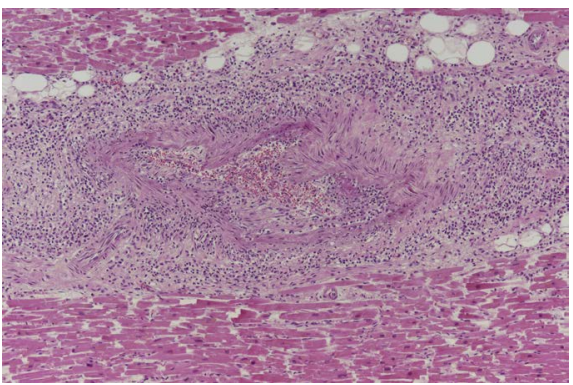

B

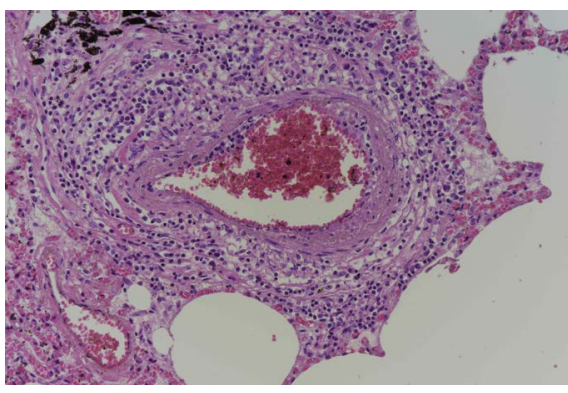

$\mathrm{E}$

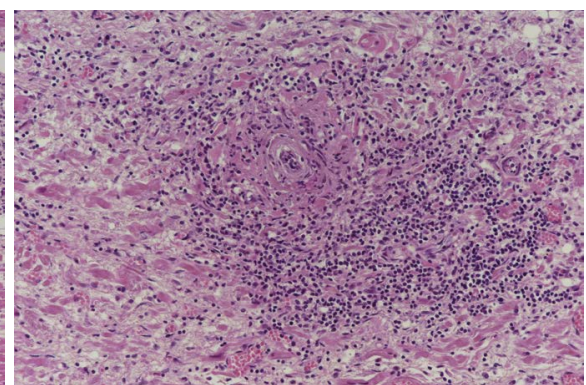

C

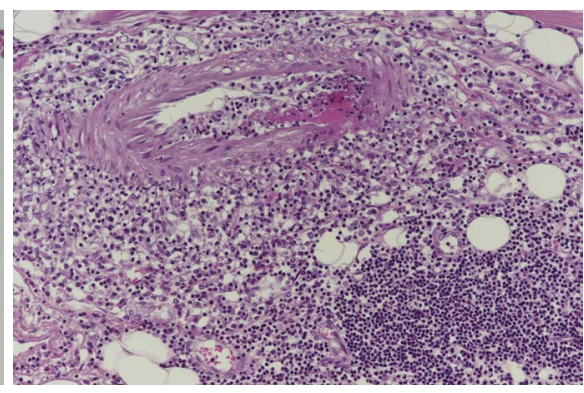

$\mathbf{F}$

Fig. 1. Vasculitis in the small vessels, infiltrated by histocytes, lymphocytes and neutrophils that extend into the perivascular soft tissue and interstitium are observed in the myocardium $(A, H \& E, \times 100 ; B, H \& E, \times 100)$ and sinoatrial node $(C, H \& E, \times 200)$. Some inflammatory infiltrates are also observed in the interstitium $(D, H \& E, \times 100)$. These findings are also observed in some small vessels in the lung $(E, H \& E, \times 200)$, and the vaccinated site of the left $\operatorname{arm}(F, H \& E, \times 200)$. 
node exhibited endotheliitis with fibrin deposition and vasculitis, infiltrated by lymphocytes, histiocytes, and neutrophils, extending into the perivascular soft tissue and surrounding fat tissue (Fig. 1AC). Some inflammatory cells were also observed in the interstitium near some areas of vasculitis, but myocarditis or myocardial necrosis were not identified (Fig. 1D). The vaccinated site of the left upper arm and some small vessels in the lung also exhibited the same histologic features of small vessel vasculitis in the myocardium (Fig. 1E, F). The epicardial coronary arteries and aorta were unremarkable. The right lobe of the thyroid revealed papillary microcarcinoma $(0.3 \mathrm{~cm}$ in diameter). Immunostaining was performed for CD4, CD8, myeloperoxidase (MPO), and CD68 on the small vessels of the myocardium (Fig. 2A-J) and vaccinated site of the left arm (Fig. 2K-0). The inflammatory cells infiltrating into the small vessels and perivascular soft tissue, comprised of histiocytes (CD68-postive), more CD4postive lymphocytes and less CD8-postivie lymphocytes, and neutrophils (MPO-positive). These features were the same in both the small myocardial vessels and the vaccinated site.

Postmortem toxicological tests revealed that antipyretic and anti-inflammatory analgesics were positive within the therapeutic levels. The CRP level in the postmortem blood was elevated $(4.28 \mathrm{ng} / \mathrm{mL}$ : reference range $<1.0 \mathrm{mg} / \mathrm{dL}$ ). The glycosylated hemoglobin level in the postmortem blood also increased (7.2\%: reference range $<5.6 \%$ ). The results of other postmortem ancillary tests including blood alcohol levels, ketone bodies, biochemistry, and thyroid function tests, the results were also unremarkable.

The cause of death was determined as sudden cardiac death due to cardiac small vessel vasculitis.

\section{Discussion}

This case illustrates an unexpected fatal case of sudden cardiac death due to cardiac small vessel vasculitis after COVID-19 vaccination (BNT162b2 nCov-19). Macroscopic examination revealed no

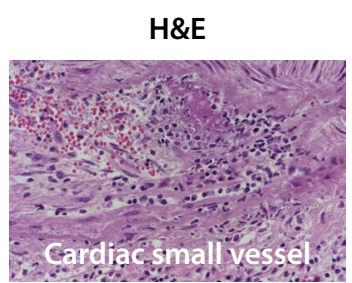

A

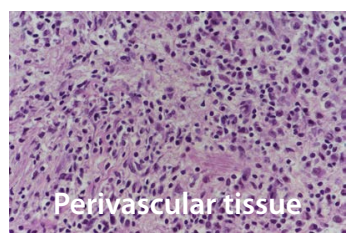

$\mathbf{F}$

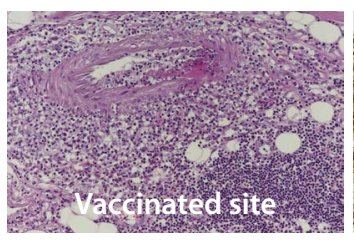

K
CD4

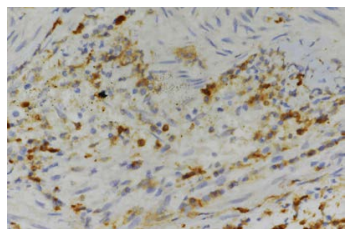

B

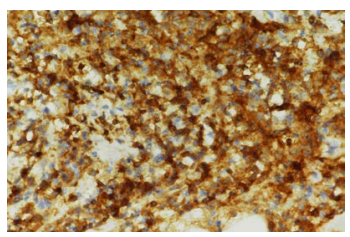

$\mathbf{G}$

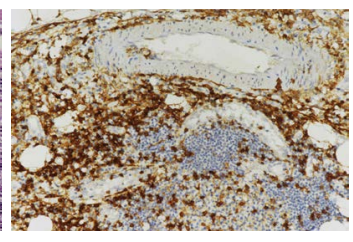

$\mathbf{L}$
CD8

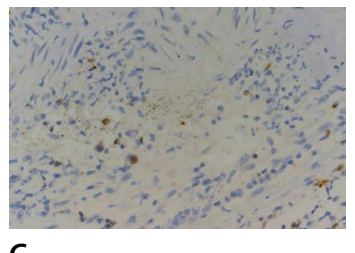

C
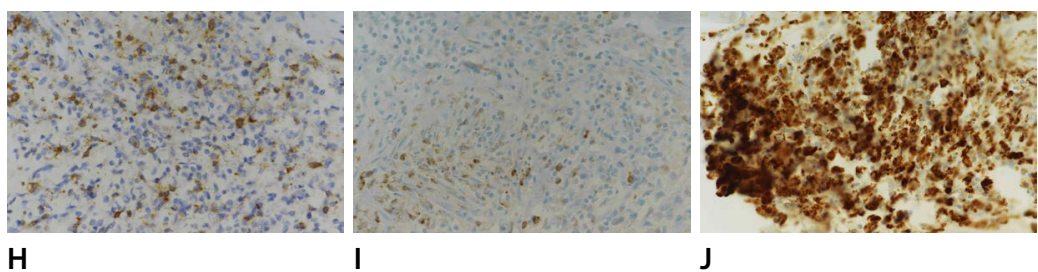

MPO

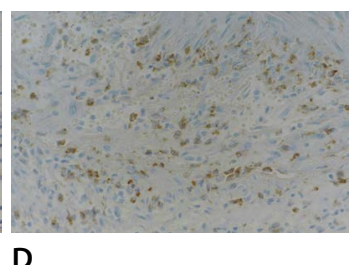

E

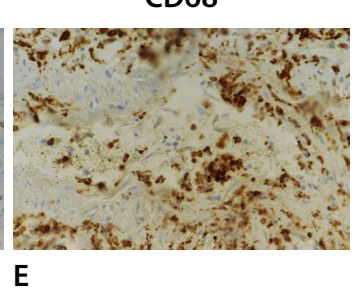

Fig. 2. Immunohistochemical study reveals that the inflammatory cells infiltrating into the small vessels $(A, H \& E, \times 400)$, perivascular area $(F$, $H \& E, \times 400)$ and vaccinated site $(K, H \& E, \times 200)$. The profiles of the inflammatory cells in these three areas have the same features that they comprise more CD4-postive lymphocytes $(B, \times 400 ; G, \times 400 ; L, \times 200)$, less CD 8-postivie lymphocytes $(C, \times 400 ; H, \times 400 ; M, \times 200)$, neutrophils (myeloperoxidase [MPO] positive; $\mathrm{D}, \times 400 ; \mathrm{I}, \times 400 ; \mathrm{N}, \times 200)$, and histiocytes (CD68 positive; $\mathrm{E}, \times 400 ; \mathrm{J}, \times 400 ; \mathrm{O}, \times 200)$. 
significant pathologic findings. Microscopically, small vessel vasculitis infiltrated by histiocytes, lymphocytes, and neutrophils was evident in the small vessels of myocardium, the injection site of vaccination, and some small vessels in the lung. Myocarditis was not observed. The clinical manifestations were progressively aggravated generalized pain for a month after vaccination along with laboratory findings of increased CRP levels. These findings suggest the possibility of systemic inflammation.

This case can be categorized as small vessel vasculitis, according to the 2012 revised International Chapel Hill Consensus Conference Nomenclature of Vasculitides [3]. However, it is difficult to provide a more specific diagnosis, as several antibody tests could not be performed during postmortem examination due to postmortem changes and degeneration, and no further clinical evaluations were performed during hospitalization. Various types of vasculitis following several vaccinations have been reported but their causal relationship could not yet be established [4]. Various infections may induce several types of vasculitis as a trigger or lead to them [4].

Myocarditis after COVID-19 vaccination (BNT162b2) and non-fatal leukocytoclastic vasculitis after mRNA COVID-19 vaccine have been reported [5-7]. In most cases of myocarditis, the patients survived, and the major clinical features were chest pain followed by fever after vaccination [5]. However, to the best of our knowledge, small vessel vasculitis in the heart after COVID-19 vaccination has not been reported yet. Given that the clinical course of small vessel vasculitis in the heart might be similar to those of myocarditis, and that vasculitis can be confirmed only by histology, some cases considered as myocarditis clinically after vaccination may be cardiac vasculitis. In cases of nonfatal leukocytoclastic vasculitis after vaccination, the major clinical findings were various cutaneous manifestations, and the patients had medical history (case 1: psoriasis, psoriatic arthritis, irritable bowel syndrome, leukocytoclastic vasculitis [6]; case 2: COVID-19 infection 3 months prior [7]). In this case, such cutaneous features were not observed.

The presence of histology-confirmed vasculitis and non-specific symptoms with increased CRP levels implying systemic inflammation in this case, are similar to those of multisystem inflammatory syndrome in adults (MIS-A) following COVID-19, characterized by fever, markedly elevated inflammatory biomarkers levels, and cardiac dysfunction [8]. The clinical and postmortem findings of an autopsy study on patients with COVID-19 and MIS were similar to those of this case (Table 1) [9]. The profile of inflammatory cell infiltrates is similar to that of moderate antibodymediated transplant rejection, suggesting that endothelial damage in COVID-19 pathogenesis may be mediated by antibody through a cytokine-storm rather than direct damage by a virus, such as in cases of antibody-mediated transplant rejection [10]. Although the deceased did not exhibit fever, it may be an atypical symptom of systemic inflammation, given that elderly patients may present atypical symptoms, including the absence of fever and new or increased confusion when they are infected [11]. In addition, it is noteworthy that the same feature of small vessel vasculitis was also observed in the vaccinated site. These findings drew the association between vasculitis and vaccination into question.

A plausible explanation for the mechanism of adverse events in COVID-19 vaccines is suggested [12]. mRNA vaccines work as immunogens and adjuvants to make target proteins (modified to stabilize the S protein; spike proteins and their fragments) by exploiting the transcription system of the host cell, and the produced proteins are rapidly recognized and the vaccinated cells are killed by the immune system $[12,13]$. The death of the host cells releases the produced proteins, and the released proteins (free-floating spike proteins) may interact with angiotensin-converting enzyme 2 receptor, which is internalized and degraded [12]. This may lead to an imbalance of the renin-angiotensin system (RAS) and be inclined to inflammation and other adverse events, which is similar to the pathogenesis of COVID-19 [12,14]. The pathologic condition and vascular dysfunction caused by diabetes mellitus and hypertension, may contribute to strengthening the proinflammatory arm of RAS and undermining the protective arm induced by the vaccine [15]. Those findings shed light on the pathogenesis of the unique findings identified in the present case. Further study 
Table 1. Clinical and postmortem findings of the COVID-19 with MIS and of this case

\begin{tabular}{lll}
\hline & \multicolumn{1}{c}{ Fox et al. [9] } & \multicolumn{1}{c}{ This case } \\
\hline Ethnicity & African American & Korean \\
Age $(\mathrm{yr})$ & 31 & 76 \\
Sex & Female & Female \\
BMI $\left(\mathrm{kg} / \mathrm{m}^{2}\right)$ & 36.1 & 32.8 \\
Medical history & Hypertension, diabetes (uncontrolled) & Hypertension, diabetes \\
Clinical manifestation & High fever, dry cough, abdominal discomfort & No fever, muscle ache, general weakness \\
& throbbing, left-sided neck pain, nausea, vomiting, & \\
Macroscopic findings & sinus tachycardia, parotitis, lymphadenopathy & \\
& Conjunctival injection, enlarged cervical and & Unremarkable \\
mediastinal lymph nodes, vascular thrombi with & \\
Microscopic findings & focal hemorrhage in left lower lung & \\
& Endotheliitis, vasculitis in small cardiac vessels, & Endotheliitis, vasculitis in cardiac small \\
& infiltrating into epicardial fat and interstitium, & vessels, infiltrating into perivascular tissue and \\
& similar inflammation in portal tracts & interstitium, similar small vessel vasculitis in
\end{tabular}

COVID-19, coronavirus diease 2019; MIS, multisystem inflammatory syndrome; BMI, body mass index; MPO, myeloperoxidase.

of additional cases is needed to establish a causal relationship between them.

In summary, we present a case of small vessel vasculitis in the heart after COVID-19 vaccination (BNT162b2), whose findings were similar to those of a COVID-19 patient with cardiac vasculitis and MIS-A. The etiology of most vasculitis cases is generally unknown, so it is difficult to clarify the causal relationship between vaccination and vasculitis. A plausible explanation for the pathogenesis of adverse events after vaccination is suggested, however, further study is needed to prove such explanation. In addition, the present case illustrates that postmortem examination is critical to confirm the cause of death. We hope that this presentation will improve understanding of the pathogenesis of small vessel vasculitis after COVID-19 vaccination.

ORCID: Sohyung Park: https://orcid.org/0000-00021987-7908; Min Kyoung Kim: https://orcid.org/00000001-9313-1103; Kanghyun Baek: https://orcid.org/00000002-5114-4384

\section{Conflicts of Interest}

No potential conflict of interest relevant to this article was reported.
Acknowledgments

This study was approved by the National Forensic Service Institutional Review Board (906-210914-BR001-01).

\section{References}

1. Polack FP, Thomas SJ, Kitchin N, et al. Safety and efficacy of the BNT162b2 mRNA Covid-19 vaccine. N Engl J Med 2020;383:2603-15.

2. Barda N, Dagan N, Ben-Shlomo Y, et al. Safety of the BNT162b2 mRNA Covid-19 vaccine in a nationwide setting. N Engl J Med 2021;385:1078-90.

3. Jennette JC, Falk RJ, Bacon PA, et al. 2012 revised International Chapel Hill Consensus Conference Nomenclature of Vasculitides. Arthritis Rheum 2013;65:1-11.

4. Bonetto C, Trotta F, Felicetti P, et al. Vasculitis as an adverse event following immunization: systematic literature review. Vaccine 2016;34:6641-51.

5. Choi S, Lee S, Seo JW, et al. Myocarditis-induced sudden death after BNT162b2 mRNA COVID-19 vaccination in Korea: case report focusing on histopathological findings. J Korean Med Sci 2021;36:e286.

6. Cohen SR, Prussick L, Kahn JS, et al. Leukocytoclastic vasculitis flare following the COVID-19 vaccine. Int J Dermatol 2021;60:1032-3.

7. Bostan E, Gulseren D, Gokoz O. New-onset leukocytoclastic vasculitis after COVID-19 vaccine. Int J Dermatol 2021;60:13056.

8. Tenforde MW, Morris SB. Multisystem inflammatory syndrome in 
adults: coming into focus. Chest 2021;159:471-2.

9. Fox SE, Lameira FS, Rinker EB, et al. Cardiac endotheliitis and multisystem inflammatory syndrome after COVID-19. Ann Intern Med 2020;173:1025-7.

10. Maccio U, Zinkernagel AS, Shambat SM, et al. SARS-CoV-2 leads to a small vessel endotheliitis in the heart. EBioMedicine 2021;63:103182.

11. van Duin D. Diagnostic challenges and opportunities in older adults with infectious diseases. Clin Infect Dis 2012;54:973-8.

12. Angeli F, Spanevello A, Reboldi G, et al. SARS-CoV-2 vaccines: lights and shadows. Eur J Intern Med 2021;88:1-8.

13. Dai L, Gao GF. Viral targets for vaccines against COVID-19. Nat Rev Immunol 2021;21:73-82.

14. Verdecchia P, Cavallini C, Spanevello A, et al. The pivotal link between ACE2 deficiency and SARS-CoV-2 infection. Eur J Intern Med 2020;76:14-20.

15. Gheblawi M, Wang K, Viveiros A, et al. Angiotensin-converting enzyme 2: SARS-CoV-2 receptor and regulator of the reninangiotensin system: celebrating the 20th Anniversary of the Discovery of ACE2. Circ Res 2020;126:1456-74. 